\title{
Evaluation of Subsurface Contact Stresses in Railroad Wheels Using an Elastic Half-Space Model
} \section{A. A. dos Santos Jr. and F. Bruni \\ Department of Mechanical Design FEM - UNICAMP \\ PO Box 6122 \\ 13083-970 Campinas, SP. Brazil carvalho@fem.unicamp.br aute@fem.unicamp.br ferbruni@sigmanet.com.br}

F. de C. Santos,

\section{T. Santos \\ Department of Applied Mathematics IMMEC - UNICAMP \\ PO Box 6065 \\ 13083-970 Campinas, SP. Brazil lucio@ime.unicamp.br}

Railroad wheels fail in two main modes: rolling surface defects like spalling, shelling and wear, and internal defects including cracks propagating after a change takes place in the original stress pattern. Although the effects of the latter are almost always catastrophic, the former is more usual. The onset of rolling surface defects depends on the strength of the surface and the applied loads. The strength is related to surface hardness and can be determined through experimental evaluation under controlled conditions. Evaluating the loads is one of the challenges for contact researchers. This paper presents the evaluation of the stress field inside elastic rolling bodies with an elliptic area of contact. This kind of model can be applied to wheel-rail contact phenomena. Typical high freight transportation loads are used in conjunction with regular recommended wheel and rail sizes. The results have shown that shear stress reaches the maximum magnitude below the surface of contact, and this explains the presence of shelling defects in service. They have also shown that a new model including plasticity is required, because the range of the stresses reached surpasses, by far, the elastic limit

Keywords: Railroad wheel stresses, contact stresses, elastic half-space

\section{Introduction}

Railroad freight car wheels are subject to mechanical and thermal loads during normal operation. The main mechanical loads are due to the weight and the driving forces. The great magnitude of the freight load is one of the main advantages that rail transportation has over other modes of land transportation, and the increase in the maximum load has been one of the challenges in applied research around the world. The driving force is usually lower than the vertical weight force. In extreme situations this load combines with other solicitations, causing bending stresses in the wheel plate. Those loads contribute to the increase in contact failures: weight loads can cause shelling on the rolling surface (tread) and driving loads can cause cracks in the wheel flange.

Thermal loads arise from the braking process. In freight cars the wheel works as a brake drum. The objective of braking is to take the kinetic and potential energy out of the train. Most of the energy is turned into heat in the regular brake system. Part of the heat flows to the wheel rim and creates a temperature gradient in the radial direction. The heated rim tends to expand and the plate, still cool, tends to restrain the rim movement. The difference in deformation between these two parts of the wheel is responsible for the rising of radial stresses in the plate. In addition, constraint causes hoop compressive stress in the rim, because expansion in the circumferential direction requires an increase in this part's mean radius. Stresses caused by braking process are called thermal stresses. A common mistake is to misconstrue the residual stresses caused by thermal stresses, sometimes wrongly called thermal stresses (Gallagher et al., 1991; Stone et al., 1992).

\section{Problems Related to Wheel Stresses}

There are three main problems related to residual stresses in the wheel: spalling, sudden fracture and shelling. Spalling is caused by the change in the material structure in small portions of the tread. This change occurs when the cool rail quenches the hot tread. Only the portion in contact has its structure changed. The original

Paper accepted October, 2004. Technical Editor: Edgar Nobuo Mamiya. structure in the tread consists of fine perlite and it can become bainite or even martensite. The train's movement and the presence of hoop tensile stresses can help small surface cracks propagate around the new structure formed, causing the piece of the material to fall off.

Sudden cracking is the most dangerous kind of problem in the wheel. The change in the original compressive stress pattern can lead to sudden cracking when the train is moving. The effects of the resulting derailment can be catastrophic. It occurs when a crack, which originated either in the tread or in another portion of the rim, propagates through a residual tensile stress field. Preventing this kind of failure is very difficult because there is no easy way to identify highly stressed wheels in the field.

Shelling is caused by contact stresses. It occurs when a surface check propagates in the direction of maximum shear stress. It happens at $45^{\circ}$ with the surface or right below the surface, parallel to it. The shells created usually disappear after few wheel revolutions because the checks are in a fine layer near the surface. Propagation of these small cracks depends on the plastic deformation in their tips and it is likely to occur at higher temperatures, like those caused by the braking process. Besides, the presence of a residual tensile stress pattern in the circumferential direction will allow those cracks to propagate deeper toward the plate, causing greater damage. This is one of the main problems in Brazilian high freight car wheels.

\section{Contact Stresses}

Contact stresses are due to weight, driving forces and other types of unusual, mainly dynamic, forces. For the simplest model to calculate the stresses, one uses the Hertz Theory and evaluates the effect of the weight only. Even more complicated theories consider the driving and other forces as a percentage of weight. So, these models require weight increase in order to include all the effects.

One of the main advantages of the railroad over other transportation modes is the amount of load it is able to carry. Brazilian railroads have been trying to increase freight cars capacity for many years. The great number of surface defects appears to be the main obstacle when one is trying to reach this goal. Railroad wheel life usually ranges from 400,000 to 1.2 million kilometers. The mean railroad wheels life is about 60 to $70 \%$ of the expected life for other countries' railroads, assuming loading characteristics 
are the same. It would appear that the real loads are higher or the wheel materials are not so strong. The wheel manufacturers are internationally recognized for the quality of their products, so the real loads should be the main cause of failures. Real loads are usually evaluated by the stresses they cause on the wheel. An incorrect formulation for stresses calculation can result in an overestimation of wheel life. It is important to get the correct values for the stress field in the contact region. This paper describes the use of analytical tools to evaluate stresses inside bodies in contact.

\section{State of the Art and Contribution}

The initial studies about the contact between two bodies took place near the end of the nineteenth century. German researcher Heinrich Hertz published one of his first papers about this subject in 1896. His studies (Hertz, 1882) were based on experiments using optical interference between two cylindrical glass lenses. He observed that both lenses deformed under load and that the contact region had an elliptic shape. When he took off the load, the contact region disappeared and the lenses reverted to their original shape. From those observations, Hertz formulated his theory that is still used by anyone who studies contact. Regardless its great importance, the Hertz Theory can be used only with perfectly elastic bodies under normal loads only. He calculated only the surface stresses.

English researcher Carter, in the late nineteen-twenties (Carter, 1926), was the first to evaluate the tangential forces between two rolling bodies. He aimed to evaluate locomotive railroad wheels in contact with the rail. He considered only the longitudinal creep, and found the tangential forces in that direction. He was also the first to propose a model to consider the creep in the longitudinal direction.

In 1949, M'Ewen (1949) evaluated the contact between two cylinders and calculated the stress field, taking into account the tangential load due the friction on the contact area. His study was the first to include the friction force on the model.

Johnson, (1985), studied the contact between two moving spheres measuring the creep coefficient in its three forms: longitudinal, transversal and spin. He also got the corresponding forces and moments. Determination of the contact region and the pressure distribution was based on the Hertz Theory. His research provided a great contribution to the understanding of contact phenomena and was documented in several papers and in a book (Johnson, 1985).

Smith and Liu (1953) studied the contact between elastic bodies with and without creep. Their model could be applied only to rectangular contact areas. They deducted the equations to calculate the normal and shear stresses analytically, both in the contact surface and inside the bodies. They used the maximum value for the tangential force, as described by Coulomb's Law. Their studies represented a big step in contact stress evaluation because they calculated the stresses under the surface, instead of only on it, like Hertz did.

In the early nineteen-sixties, Haines and Ollerton (1963) came up with an approximated solution for shear stress distribution in an elliptic contact area. They also compared their results with photo elasticity experiments and found good agreement.

Hamilton and Goodman (1966) found, analytically, the stress field for a circular contact area, both on the surface and under it. They also used Hertz Theory and included the sliding effect using Coulomb's Law.

In the late nineteen-sixties, Kalker (1967) presented his Ph.D. thesis in which he proposed a new revolutionary theory for contact between rolling bodies. He calculated all tangential forces and creep coefficients. He also found the rigidity parameters involved in the analysis. His work was also based on the Hertz Theory. He used it to develop several simplified algorithms (FASTSIM) and complete programs (CONTACT) to calculate the forces in the contact. His work provided an outstanding contribution to railroad research, because his theory explained and allowed one to calculate several parameters used in the wheel-rail modeling, like creep, yaw angle, spin and others.

Sackfield and Hills (1983) put together the existing theories for circular and rectangular contact areas. They considered both the longitudinal and tangential loads. They proposed a model to obtain the stress field for both cases; each one was identified using the axle ratio $k$. The results they got were similar to the ones calculated by other researchers before them.

The main contribution of this work is its application to the wheel/rail contact problem, as a means to study a type of failure that is commonplace in railroad wheels, which originate from the stresses created at the contact interface: shelling. Basically, the authors we quote have calculated subsurface stresses considering the contact between two spheres or two cylinders, which creates a circular or rectangular contact area, respectively. With these shapes, the contact area dimensions are determined analytically, by solving simple equations. Subsurface stresses may also be found by means of purely analytical expressions. When we work with a more complex contact, such as wheel / rail contact, the contact area becomes elliptic, since the bodies' curvature is different and they are in perpendicular plans. For the case of elliptic contact, the equations cannot be solved in a trivial manner; numeric analysis is required. The determination of the contact ellipse's semi-axles is performed by means of a semi-analytical process, in order to solve elliptic integrals. The same situation occurs with the subsurface stresses. The stresses' expressions require the resolution of a double integral that does not exist in a primitive manner. Thus, one must resort to the use of numeric methods for the stresses' calculation. With the results obtained through this work, it is possible to calculate the distribution of the stresses resulting in the vicinity of the contact, which may allow comparison with proper resistance models, in future studies.

\section{Objective}

The objective of this work is to find the stress tensor in any place of wheels and rails near the contact region, as a part of a study to evaluate wheel and rail life. An elastic half-space model describes the contact region and Boussineq and Cerruti potential functions are used together with Hooke's law to find the displacements that are used to calculate the stress field. Three contact area shapes are analyzed: circular, rectangular and elliptic. The Gauss-Lobato method is used to solve the final equations. The results will be used for the stresses' evaluation in a typical loading condition, in keeping with the characteristics of the Brazilian railroads that carry the heaviest loads. Specifically we will work with nominal data related to iron-ore carrying railway cars belonging to Estrada de Ferro Carajás, a subsidiary of Companhia Vale do Rio Doce, with a total load of 120 tons on eight wheels.

\section{Displacements by Boussinesq and Cerruti Potential Functions}

Evaluating surface and subsurface stresses requires the calculation of the displacements field. One also needs to calculate the contact area. The displacements can be found using the Boussinesq and Cerruti potential functions and the Theory of Elasticity. The results are calculated using only one normal force, not the tangential or longitudinal ones. It is assumed that an elastic half-space model can describe the contact, i.e., one should consider only the contact region without taking its dimensions into account. It 
means that the model assumes that the bodies are elastic and their dimensions are much greater than the contact region. The loads are two-dimensional and the stress field is plotted in three dimensions. So, the symmetric stress tensor has six components:

$$
\sigma_{i j}\left(\begin{array}{ccc}
\sigma_{x} & \tau_{\mathrm{xy}} & \tau_{\mathrm{xz}} \\
& \sigma_{y} & \tau_{\mathrm{yz}} \\
\operatorname{sim} & & \sigma_{\mathrm{z}}
\end{array}\right)
$$

The classical modeling to find a stress and displacement fields was presented by Boussinesq and Cerruti. They used the potential theory. This modeling is presented by Love (1929). A solid model is called an elastic half-space when all of its points are placed on just one side of a " $z$ " plane which meets the requirements of the following relationship: $\{(x, y, z): z \geq 0\}$.

Considering the half-space model shown in Fig. 1, one could define an arbitrary position described by $C(\xi, \eta)$, inside the contact area $S$, and another arbitrary position described by $A(x, y, z)$ subsurface of the bodies. Consider the distance $R$ as the module of the position of point $A$ related to point $C$, as shown in Eq.(2).

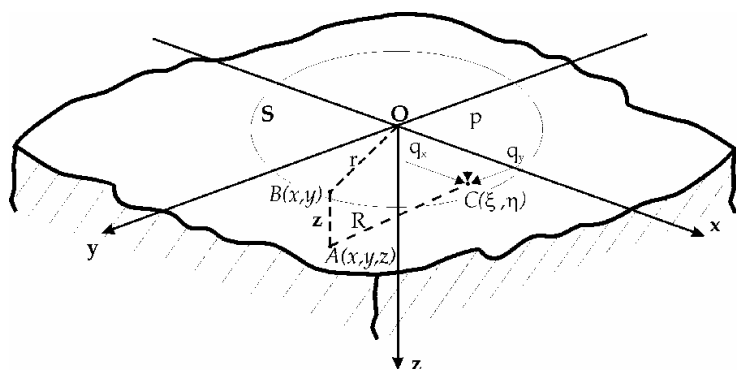

Figure 1. Elastic half-space model.

$$
R=\left((\xi-x)^{2}+(\eta-y)^{2}+z\right)^{1 / 2}
$$

The $p_{z}(\xi, \eta)$ function represents the pressure distribution on the contact surface and $q_{x}(\xi, \eta)$ and $q_{y}(\xi, \eta)$ represent the tangential stress distribution in the $x$ and $y$ direction, respectively. The potential functions $F_{1}, G_{1}$ and $H_{1}$ can be defined by Eqs. (3), (4) and (5) These functions represent the total load applied in one direction $(x, y, z)$. The functions also meet the requirements of Laplace's equation.

$$
\begin{aligned}
& F_{1}=\iint_{S} q_{x}(\xi, \eta) \Omega d \xi d \eta \\
& G_{1}=\iint_{S} q_{y}(\xi, \eta) \Omega d \xi d \eta \\
& H_{1}=\iint_{S} P_{z}(\xi, \eta) \Omega d \xi d \eta
\end{aligned}
$$

Where $\Omega$ is a harmonic variable used to reduce previous equations (Love, 1929). It is given by:

$$
\Omega=z \ln (R+z)-R
$$
(9)

The derivative of the potential function is shown in Eqs. (7) to 9).

$$
\begin{aligned}
& F=\frac{\partial F_{1}}{\partial z}=\iint_{S} q_{x}(\xi, \eta) \ln (R+z) d \xi d \eta \\
& G=\frac{\partial G_{1}}{\partial z}=\iint_{S} q_{y}(\xi, \eta) \ln (R+z) d \xi d \eta \\
& H=\frac{\partial H_{1}}{\partial z}=\iint_{S} P_{z}(\xi, \eta) \ln (R+z) d \xi d \eta
\end{aligned}
$$

From potential functions one can define the parameters $\phi$ and $\phi_{1}$, that are harmonic functions of $\mathrm{x}, \mathrm{y}$ and $\mathrm{z}$. So they have to satisfy Laplace's Equation, as described in Eqs. (10) to (13).

$$
\begin{gathered}
\nabla^{2} \phi_{1}=0 \\
\nabla^{2} \phi=0 \\
\phi_{1}=\frac{\partial F_{1}}{\partial x}+\frac{\partial G_{1}}{\partial y}+\frac{\partial H_{1}}{\partial z} \\
\phi=\frac{\partial \phi_{1}}{\partial z}=\frac{\partial F}{\partial x}+\frac{\partial G}{\partial y}+\frac{\partial H}{\partial z}
\end{gathered}
$$

Hertz (1882) and Boussinesq (in love, 1929) deducted the $u_{x}, u_{y}$ and $u_{z}$ components of the elastic displacement in an arbitrary position $A(x, y, z)$ by using the potential functions. The relations are shown in Eqs. (14) to (16).

$$
\begin{gathered}
u_{x}=\frac{1}{4 \pi G}\left\{2 \frac{\partial F}{\partial z}-\frac{\partial H}{\partial x}+2 v \frac{\partial \phi_{1}}{\partial x}-z \frac{\partial \phi}{\partial x}\right\} \\
u_{y}=\frac{1}{4 \pi G}\left\{2 \frac{\partial G}{\partial z}-\frac{\partial H}{\partial y}+2 v \frac{\partial \phi_{1}}{\partial y}-z \frac{\partial \phi}{\partial y}\right\} \\
u_{z}=\frac{1}{4 \pi G}\left\{2 \frac{\partial H}{\partial z}+(1-2 v) \phi-z \frac{\partial \phi}{\partial z}\right\}
\end{gathered}
$$

Considering only the normal stresses, i.e., the pressure distribution $p_{z}(\xi, \eta)$ caused by vertical forces, the friction forces are null and the values of $q_{x}(\xi, \eta)$ and $q_{y}(\xi, \eta)$ vanish, as shown in Eq.(17).

$$
F=F_{1}=G=G_{1}=0
$$

With this simplification, the harmonic functions can be reduced to:

$$
\begin{gathered}
\phi_{1}=\frac{\partial H_{1}}{\partial z}=H=\iint_{S} P_{z}(\xi, \eta) \ln (R+z) d \xi d \eta \\
\phi_{1}=\frac{\partial H}{\partial z}=\frac{\partial \phi_{1}}{\partial z}=\iint_{S} P_{z}(\xi, \eta) \frac{1}{R} d \xi d \eta
\end{gathered}
$$

Taking Eqs. (18), (19) and Eqs. (14) to (16), results: 


$$
\begin{aligned}
& u_{x}=-\frac{1}{4 \pi G}\left\{(1-2 v) \frac{\partial \phi_{1}}{\partial x}+z \frac{\partial \phi}{\partial x}\right\} \\
& u_{y}=-\frac{1}{4 \pi G}\left\{(1-2 v) \frac{\partial \phi_{1}}{\partial y}+z \frac{\partial \phi}{\partial y}\right\} \\
& u_{z}=-\frac{1}{4 \pi G}\left\{2(1-2 v) \phi-z \frac{\partial \phi}{\partial z}\right\}
\end{aligned}
$$

In the previous equation, $G$ is the shear modulus and $v$ is the Poisson coefficient.

\section{Modeling Elliptic Contact Areas}

The wheel-rail contact can be described by the general case of an elliptic contact area. Although there are some important simplifications that can be applied in modeling other formats of the contact areas, these will not be developed here. The circular contact area is important for roller bearings studies and the rectangular contact area is important for modeling contact in roller bearings and gears.

\section{Pressure Applied to an Elliptic Contact Region}

Considering the $a$ and $b$ semi-axles of the ellipse formed in the contact region of two bodies with arbitrary curvature, the pressure distribution has the shape described as follows (Hertz, 1882):

$$
p_{z}(x, y)=p_{0}\left\{1-(x / a)^{2}-(y / b)^{2}\right\}^{1 / 2}
$$

This pressure acts on the elliptic region described by:

$$
(x / a)^{2}+(y / b)^{2}-1=0
$$

Using the classical model with the Boussinesq potential functions, Eq.(19) can be written as follows:

$$
\phi(x, y, z)=\iint_{S}\left\{1-(\xi / a)^{2}-(\eta / b)^{2}\right\}^{1 / 2} R^{-1} d \xi d \eta
$$

The component normal to surface of the displacement can be calculated through Eq.(22) using $z=0$, as shown.

$$
\bar{u}_{z}(x, y)=\frac{1-v}{2 \pi G}(\phi)_{z=0}
$$

Using the potential theory for one arbitrary position inside one of the bodies (Love, 1929):

$$
\begin{aligned}
& \phi(x, y, z)=\frac{1}{2} \pi p_{0} a b \int_{\lambda_{1}}^{\infty}\left(1-\frac{x^{2}}{a^{2}+w}-\frac{y^{2}}{b^{2}+w}-\frac{z^{2}}{w}\right) \times \\
& \frac{d w}{\left\{\left(a^{2}+w\right)\left(b^{2}+w\right) w\right\}^{1 / 2}}
\end{aligned}
$$

In the previous equation, $\lambda_{1}$ is the greatest positive root of the Eq.(28).

$$
\frac{x^{2}}{a^{2}+\lambda}+\frac{y^{2}}{b^{2}+\lambda}+\frac{z^{2}}{\lambda}=1
$$

Analyzing just the surface $(z=0)$, one can get:

$$
\begin{aligned}
& \phi(x, y, 0)=\frac{1}{2} \pi p_{0} a b \int_{0}^{\infty}\left(1-\frac{x 2}{a^{2}+w}-\frac{y^{2}}{b^{2}+w}\right) \times \\
& \left\{\left(a^{2}+w\right)\left(b^{2}+w\right) w\right\}^{1 / 2}
\end{aligned}
$$

So, the displacement in the surface of the elliptic region is:

$$
\bar{u}_{x}=\frac{1-v^{2}}{\pi E}\left(L-M x^{2}-N y^{2}\right)
$$

In the previous equation, $\mathrm{M}, \mathrm{N}$ and $\mathrm{L}$ are given by:

$$
\begin{aligned}
& M=\frac{\pi p_{0} a b}{2} \int_{0}^{\infty} \frac{d w}{\left\{\left(a^{2}+w\right)^{3}\left(b^{2}+w\right) w\right\}^{1 / 2}} \\
& =\frac{\pi p_{0} b}{e^{2} a^{2}}\{K(e)-E(e)\} \\
& N=\frac{\pi p_{0} a b}{2} \int_{0}^{\infty} \frac{d w}{\left\{\left(a^{2}+w\right)\left(b^{2}+w\right)^{3} w\right\}^{1 / 2}} \\
& =\frac{\pi p_{0} b}{e^{2} a^{2}}\left\{\frac{a^{2}}{b^{2}} E(e)-K(e)\right\} \\
& L=\frac{\pi p_{0} a b}{2} \int_{0}^{\infty} \frac{d w}{\left\{\left(a^{2}+w\right)\left(b^{2}+w\right) w\right\}^{1 / 2}} \\
& =\pi p_{0} b K(e)
\end{aligned}
$$

In the previous equations $E(e)$ and $K(e)$ are complete elliptic integrals and $e$ the eccentricity given by:

$$
e=\left(1-b^{2} / a^{2}\right)^{1 / 2}
$$

\section{Geometric Constants}

The calculation of the contact areas requires knowledge of some geometric constants used in the formulation. Consider Fig. 2, where two solid discs are put together to generate an elliptic contact area. The maximum curvature radius for body 1 is $R^{\prime}{ }_{1}$ and the minimum is $R_{l}$. The same notation is used for body 2 . The coordinate systems $\left(U_{1}, V_{1}\right)$ and $\left(U_{2}, V_{2}\right)$, attached to each body, have an angle of $a$ between them. Initially, the contact between the bodies is in just one point. Considering $z$ the distance of two points in the surfaces near the contact point, measured perpendicular to the contact areas, it can be calculated by:

$$
z-A x^{2}+B y^{2}
$$

$\mathrm{A}$ and $\mathrm{B}$ are geometric constants, which depends on the curvature of the bodies and the position $\mathrm{x}$ and $\mathrm{y}$ with regard to the 
coordinate axles $\mathrm{X}$ and $\mathrm{Y}$, in the tangent common plane. Assuming that Eq.(35) describes the contact area, it is elliptical. Figure 3 shows part of body 1 and the $z_{1}$ distance, which is measured from the tangent plane to some arbitrary point $\mathrm{C}$ or $\mathrm{D}$ in the surface of the same body and near the contact point.

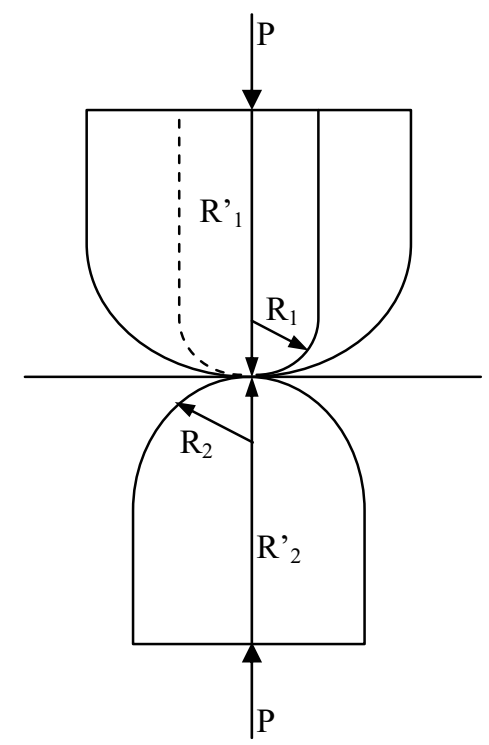

Figure 2. Scheme of two solids pressed together.
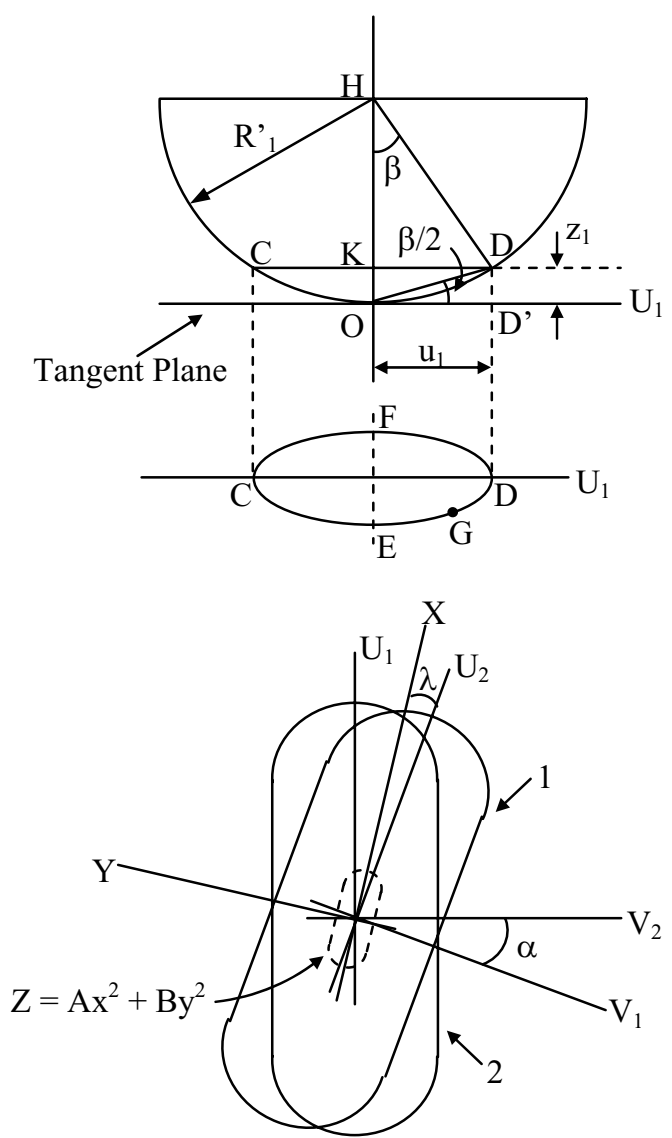

Figure 3. Deformations in the contact surface.
If the contact is local in nature, the $z_{1}$ distance can be found using the ODD' triangle:

$$
z_{1}=u_{1} \tan \left(\frac{1}{2} \beta\right)=\frac{1}{2} u_{1} \beta
$$

Considering small $\beta$ and $\mathrm{R}_{1}$ approximately equal to $\mathrm{HK}$, from HKD triangle:

$$
\tan \beta=\beta=\frac{K D}{H K}=\frac{u_{1}}{R_{1}^{\prime}}
$$

Replacing Eqs. (37) in (36) results in:

$$
z_{1}=\frac{u_{1}^{2}}{R_{1}^{\prime}}
$$

Likewise, the $\mathrm{z}_{1}$ distance from contact center to point $\mathrm{E}$ or $\mathrm{F}$, which are on the same plane as $R_{1}$ is:

$$
z_{1}=\frac{v_{1}^{2}}{2 R_{1}^{\prime}}
$$

The distance to an arbitrary point $\mathrm{G}$, which is not in the main axles, is the sum of both $z_{1}$ distances described by Eqs. (38) and (39). This sum is an elliptic equation, expressed by:

$$
z_{1}=\frac{u_{1}^{2}}{R_{1}^{\prime}}+=\frac{v_{1}^{2}}{2 R_{1}}
$$

Body 2 can be analyzed using the same steps and the $\mathrm{z}_{2}$ distance can be described as:

$$
z_{2}=\frac{u_{2}^{2}}{R_{2}^{\prime}}+=\frac{v_{2}^{2}}{2 R_{2}}
$$

The total $\mathrm{z}$ distance is the sum of $\mathrm{z}_{1}$ and $\mathrm{z}_{2}$ :

$$
z=z_{1}+z_{2}=\frac{u_{1}^{2}}{2 R_{1}^{\prime}}+\frac{v_{1}^{2}}{2 R_{1}}+\frac{u_{2}^{2}}{R_{2}^{\prime}}+\frac{v_{2}^{2}}{2 R_{2}}
$$

To find the geometric constants shown in Eq.(35), one must put Eq.(42) in the same format. The step is vanish $\mathrm{u}_{2}$ and $\mathrm{v}_{2}$ using the geometric relations:

$$
\begin{aligned}
& u_{2}=u_{1} \cos \alpha+v_{1} \operatorname{sen} \alpha \\
& v_{2}=u_{1} \operatorname{sen} \alpha+v_{1} \cos \alpha
\end{aligned}
$$

Replacing Eqs. (43) and (44) in (42), results in:

$$
z=A^{\prime} u_{1}^{2}+2 H^{\prime} u_{1} v_{1}+B^{\prime} v_{1}^{2}
$$

where:

$$
2 A^{\prime}=\left(\frac{1}{R_{1}^{\prime}}\right)+\left(\frac{1}{R_{2}^{\prime}}\right) \cos ^{2} \alpha+\left(\frac{1}{R_{2}}\right) \operatorname{sen}^{2} \alpha
$$




$$
\begin{gathered}
2 H^{\prime}=\left(\frac{1}{R_{2}^{\prime}}\right)-\left(\frac{1}{R_{2}}\right) \operatorname{sen} \alpha \cos \alpha \\
2 B^{\prime}=\left(\frac{1}{R_{1}}\right)+\left(\frac{1}{R_{2}^{\prime}}\right) \operatorname{sen}^{2} \alpha+\left(\frac{1}{R_{2}}\right) \cos ^{2} \alpha
\end{gathered}
$$

Changing the coordinate systems from $\left(\mathrm{U}_{1}, \mathrm{~V}_{1}\right)$ to $(\mathrm{X}, \mathrm{Y})$ requires the use of the following relations:

$$
\begin{aligned}
& u_{1}=x \cos \lambda-y \operatorname{sen} \lambda \\
& v_{1}=x \operatorname{sen} \lambda+y \cos \lambda
\end{aligned}
$$

Replacing the previous relations in Eq.(45) gives Eq.(35), where the geometric constants are:

$$
\begin{aligned}
& A=\frac{1}{R_{1}}+\frac{1}{R_{2}}+\frac{1}{R_{1}^{\prime}}+\frac{1}{R_{2}^{\prime}}- \\
& \frac{1}{4}\left(\begin{array}{l}
{\left[\left(\frac{1}{R_{1}}-\frac{1}{R_{1}^{\prime}}\right)+\left(\frac{1}{R_{2}}-\frac{1}{R_{2}^{\prime}}\right)\right]^{2}-} \\
4\left(\frac{1}{R_{1}}-\frac{1}{R_{1}^{\prime}}\right)\left(\frac{1}{R_{2}}-\frac{1}{R_{2}^{\prime}}\right) \operatorname{sen}^{2} \alpha
\end{array}\right)^{1 / 2} \\
& B=\frac{1}{4}\left(\frac{1}{R_{1}}+\frac{1}{R_{2}}+\frac{1}{R_{1}^{\prime}}+\frac{1}{R_{2}^{\prime}}\right)+ \\
& \frac{1}{4}\left(\begin{array}{l}
{\left[\left(\frac{1}{R_{1}}-\frac{1}{R_{1}^{\prime}}\right)+\left(\frac{1}{R_{2}}-\frac{1}{R_{2}^{\prime}}\right)\right]^{2}-} \\
4\left(\frac{1}{R_{1}}-\frac{1}{R_{1}^{\prime}}\right)\left(\frac{1}{R_{2}}-\frac{1}{R_{2}^{\prime}}\right) \operatorname{sen}^{2} \alpha
\end{array}\right)^{1 / 2}
\end{aligned}
$$

Figure 4 shows the relation between the displacements of the body centers and the displacements and deformation in an arbitrary point in the contact region. From that figure, one can get the relation:

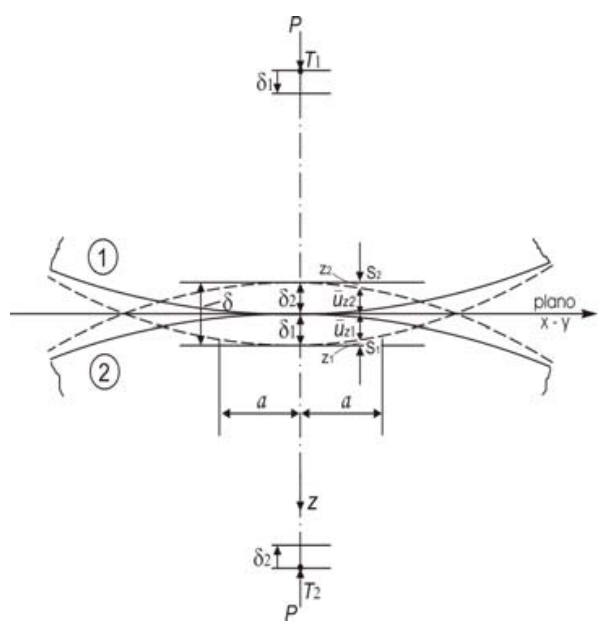

Figure 4. Displacements of two solids in contact.

$$
\begin{aligned}
& \delta-z=\bar{u}_{z 1}+\bar{u}_{z 2} \Rightarrow \\
& \delta-A x^{2}-B y^{2}=\bar{u}_{z 1}+\bar{u}_{z 2}
\end{aligned}
$$

\section{Elliptic Contact Area}

Equation (30) shows the displacement caused by the pressure distribution. Using the elastic relation from Eq.(53), one can express the sum of the displacements.

$$
\delta-A x^{2}-B y^{2}=\frac{1}{\pi E^{*}}\left(L-M x^{2}-N y^{2}\right)
$$

Equating Eq. (30) and (53), one can find that the geometric constants have the following values:

$$
A=\frac{M}{p E^{*}} \quad B=\frac{N}{p E^{*}} \quad d=\frac{L}{p E^{*}}
$$

Using Eq. (31) to (33) to compare with previous equation, one can find:

$$
\begin{gathered}
A=\frac{p_{0}}{E^{*}} \frac{b}{e^{2} a^{2}}\{K(e)-E(e)\} \\
B=\frac{p_{0}}{E^{*}} \frac{b}{e^{2} a^{2}}\left\{\frac{a^{2}}{b^{2}} E(e)-K(e)\right\} \\
\delta=\frac{p_{0}}{E^{*}} b K(e)
\end{gathered}
$$

The semi-axles $a$ and $b$ of the ellipse can be found by:

$$
\frac{B}{A}=\left(\frac{a}{b}\right)^{2} \frac{E(e)-K(e)}{K(e)-E(e)}
$$

The process to get $a$ and $b$ depends on the eccentricity, which depends on $a$ and $b$ as described by means of Eq.(60) equation. So, an interactive process is required to find the ellipse axles.

$$
e=\left(1-b^{2} / a^{2}\right)^{1 / 2} \quad b<a
$$

\section{Stresses in Elliptic Contact Areas}

The generalized Hooke's Law expresses the stress-strain relation in an orthogonal $(\mathrm{x}, \mathrm{y}, \mathrm{z})$ system as:

$$
\begin{aligned}
& \varepsilon_{x}=\frac{1}{E}\left[\sigma_{x}-v\left(\sigma_{y}+\sigma_{z}\right)\right] \\
& \varepsilon_{y}=\frac{1}{E}\left[\sigma_{y}-v\left(\sigma_{x}+\sigma_{z}\right)\right] \\
& \varepsilon_{z}=\frac{1}{E}\left[\sigma_{z}-v\left(\sigma_{x}+\sigma_{y}\right)\right]
\end{aligned}
$$

In the previous $\varepsilon_{x}, \varepsilon_{y}, \varepsilon_{z}$ e $\sigma_{x}, \sigma_{y}, \sigma_{z}$ are strains and stresses in the $(\mathrm{x}, \mathrm{y}, \mathrm{z})$ coordinates, respectively. The stresses can be calculated inverting those equations: 


$$
\begin{aligned}
& \sigma_{x}=\frac{E \varepsilon_{x}(1-v)+v E\left(\varepsilon_{y}+\varepsilon_{z}\right)}{1-v-2 v^{2}} \\
& \sigma_{y}=\frac{E \varepsilon_{y}(1-v)+v E\left(\varepsilon_{x}+\varepsilon_{z}\right)}{1-v-2 v^{2}} \\
& \sigma_{z}=\frac{E \varepsilon_{z}(1-v)+v E\left(\varepsilon_{x}+\varepsilon_{y}\right)}{1-v-2 v^{2}}
\end{aligned}
$$

The strains can be found by the derivative of displacements:

$$
\varepsilon_{x}=\frac{\partial u_{x}}{\partial x} \quad \varepsilon_{y}=\frac{\partial u_{y}}{\partial y} \quad \varepsilon_{z}=\frac{\partial u_{z}}{\partial z}
$$

Merging Eq.(64) to (66) with Eq.(67) yields:

$$
\begin{gathered}
\sigma_{x}=\frac{2 v G}{1-2 v}\left(\frac{\partial u_{x}}{\partial x}+\frac{\partial u_{y}}{\partial y}+\frac{\partial u_{z}}{\partial z}\right)+2 G \frac{\partial u_{x}}{\partial x} \\
\sigma_{x}=\frac{2 v G}{1-2 v}\left(\frac{\partial u_{x}}{\partial x}+\frac{\partial u_{y}}{\partial y}+\frac{\partial u_{z}}{\partial z}\right)+2 G \frac{\partial u_{y}}{\partial y} \\
\sigma_{x}=\frac{2 v G}{1-2 v}\left(\frac{\partial u_{x}}{\partial x}+\frac{\partial u_{y}}{\partial y}+\frac{\partial u_{z}}{\partial z}\right)+2 G \frac{\partial u_{z}}{\partial z}
\end{gathered}
$$

Also, one can get the shear stresses using the same procedure:

$$
\begin{aligned}
& \tau_{x y}=G\left(\frac{\partial u_{x}}{\partial y}+\frac{\partial u_{y}}{\partial x}\right) \\
& \tau_{x z}=G\left(\frac{\partial u_{x}}{\partial z}+\frac{\partial u_{z}}{\partial x}\right) \\
& \tau_{y z}=G\left(\frac{\partial u_{y}}{\partial z}+\frac{\partial u_{z}}{\partial y}\right)
\end{aligned}
$$

The expression for stresses using just normal load, as decided previously, are related to the expansion concept from Elasticity Theory. This concept can be expressed by:

$$
\frac{\partial u_{x}}{\partial x}+\frac{\partial u_{y}}{\partial y}+\frac{\partial u_{z}}{\partial z}=\frac{1-2 v}{2 \pi G} \frac{\partial \phi}{\partial z}
$$

The replacement of Eq.(74) in the equations for stress calculation yields:

$$
\begin{aligned}
& \sigma_{x}=\frac{1}{2 \pi}\left(2 v \frac{\partial \phi}{\partial z}-z \frac{\partial^{2} \phi}{\partial x^{2}}-(1-2 v)\right) \frac{\partial^{2} \phi_{1}}{\partial x^{2}} \\
& \sigma_{y}=\frac{1}{2 \pi}\left(2 v \frac{\partial \phi}{\partial z}-z \frac{\partial^{2} \phi}{\partial y^{2}}-(1-2 v)\right) \frac{\partial^{2} \phi_{1}}{\partial y^{2}}
\end{aligned}
$$

$$
\begin{gathered}
\sigma_{z}=\frac{1}{2 \pi}\left(\frac{\partial \phi}{\partial z}-z \frac{\partial^{2} \phi}{\partial z^{2}}\right) \\
\sigma_{z}=\frac{1}{2 \pi}\left(\frac{\partial \phi}{\partial z}-z \frac{\partial^{2} \phi}{\partial z^{2}}\right) \\
\tau_{x y}=-\frac{1}{2 \pi}(1-2 v) \frac{\partial^{2} \phi_{1}}{\partial x \partial y}+z \frac{\partial^{2} \phi}{\partial x \partial y}
\end{gathered}
$$

$$
\tau_{x z}=\frac{1}{2 \pi}\left(\frac{\partial^{2} \phi}{\partial x \partial z}\right)
$$

$$
\tau_{x z}=\frac{1}{2 \pi}\left(\frac{\partial^{2} \phi}{\partial x \partial z}\right)
$$

$$
\tau_{x z}=\frac{1}{2 \pi}\left(\frac{\partial^{2} \phi}{\partial y \partial z}\right)
$$

Equations (75) to (80) equations yield the stress field to the contact region in elastic bodies without friction.

\section{Results and Discussion}

The equations developed make it possible to determine the distribution of the stresses in the contact area in any body that has an elliptic contact area. In order to study the shelling issue in railroad wheels and rails - both manufactured in steel - one must determine the elastic and geometric properties, in addition to the load applied. Table 1 shows the elastic properties used and the curvature radiuses of both bodies. Both in the wheel and in the rail, these radiuses are on perpendicular planes. The wheel's shape on the plane on which the rail is curved is a straight line (infinite radius), tangential to the rail's curvature. The same situation occurs with the rail on the plane on which the wheel is curved.

The results shown take into consideration a coordinates system with the " $x$ " axle parallel to the contact plane, in the wheel's rolling direction; the "y" axle is parallel to the contact plane and its direction is perpendicular to the " $x$ " axle; the " $\mathrm{z}$ " axle direction is perpendicular to the contact plane.

Figures 5 to 10 show the plots for stresses along the $\mathrm{z}$ coordinated axle, where the stresses have maximum magnitudes. The maximum values for normal stresses are on the surface, coherently, and those are also along the z-coordinate. The maximum values for shear stresses are found right below the surface. As reported before, it is the main cause of the shelling problems reported by Brazilian Railroads (Santos, 2000).

The results shown take into consideration a coordinates system with the " $x$ " axle parallel to the contact plane, in the wheel's rolling direction; the " $y$ " axle is parallel to the contact plane and its direction is perpendicular to the " $\mathrm{x}$ " axle; the " $\mathrm{z}$ " axle direction is perpendicular to the contact plane.

Figures 5 to 10 show the plots for stresses along the zcoordinated axle, where the stresses have maximum magnitudes. The maximum values for normal stresses are on the surface, coherently, and those are also along the z-coordinate. The maximum values for shear stresses are found right below the surface. As reported before, it is the main cause of the shelling problems reported by Brazilian Railroads (Santos, 2000). 
Table 1. Outline Conditions for the Calculation of Wheel/Rail Contact Stresses.

\begin{tabular}{|c|c|c|c|c|c|}
\cline { 2 - 5 } \multicolumn{1}{c|}{} & $\begin{array}{c}\text { Elasticity } \\
\text { Modulus } \\
\text { E }(\mathrm{MPa})\end{array}$ & $\begin{array}{c}\text { Poisson's } \\
\text { Coefficient } \\
\mathrm{v}\end{array}$ & $\begin{array}{c}\text { Minimum } \\
\text { Radius } \\
\mathrm{R}(\mathrm{mm})\end{array}$ & $\begin{array}{c}\text { Maximum } \\
\text { Radius } \\
\mathrm{R}^{\prime}(\mathrm{mm})\end{array}$ & $\begin{array}{c}\text { Loading } \\
\mathrm{F}(\mathrm{N})\end{array}$ \\
\hline Wheel & 207000 & 0,3 & 483 & $\infty$ & $\begin{array}{c}150000 / \\
\text { wheel }\end{array}$ \\
\hline Rail & 207000 & 0,3 & 254 & $\infty$ & $\infty$ \\
\hline
\end{tabular}

$$
\square_{x}(M P a)
$$

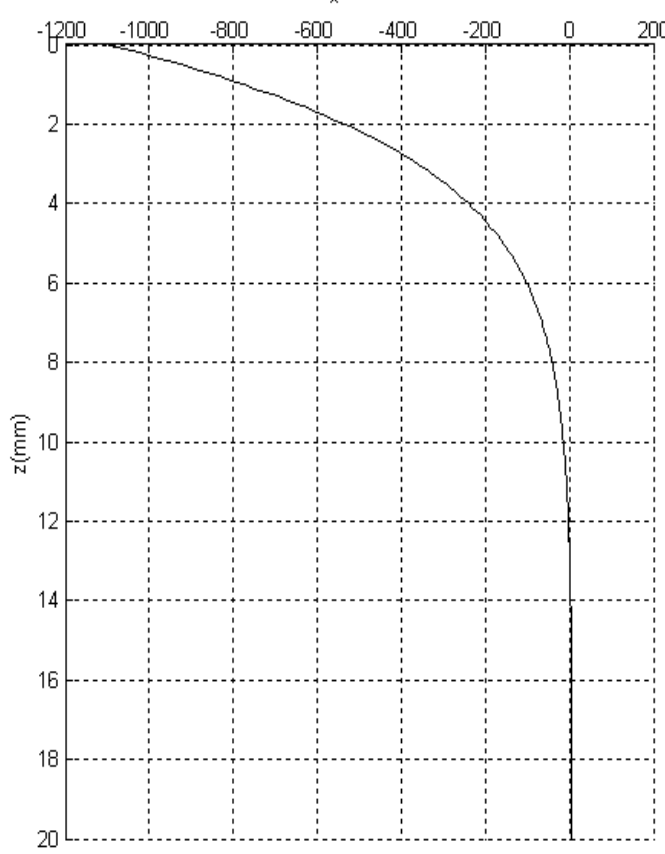

Figure 5. Normal Stress $\sigma x$ along the z-coordinated axle.

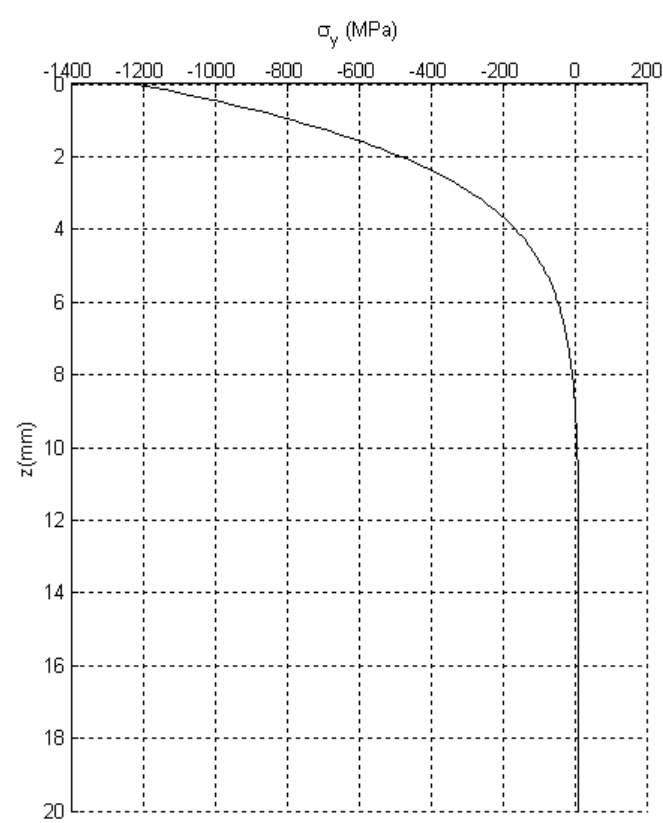

Figure 6. Normal Stress $\sigma y$ along the z-coordinated axle.

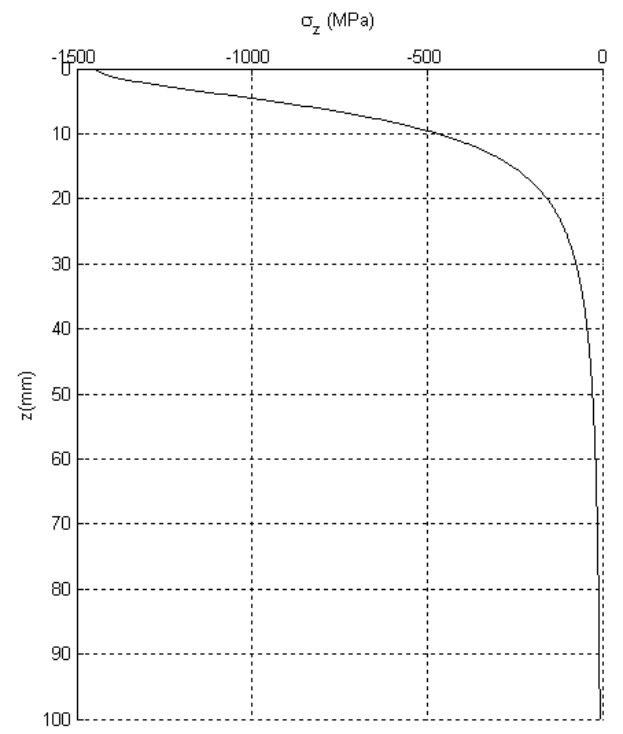

Figure 7. Normal Stress $\sigma z$ along the z-coordinated axle.

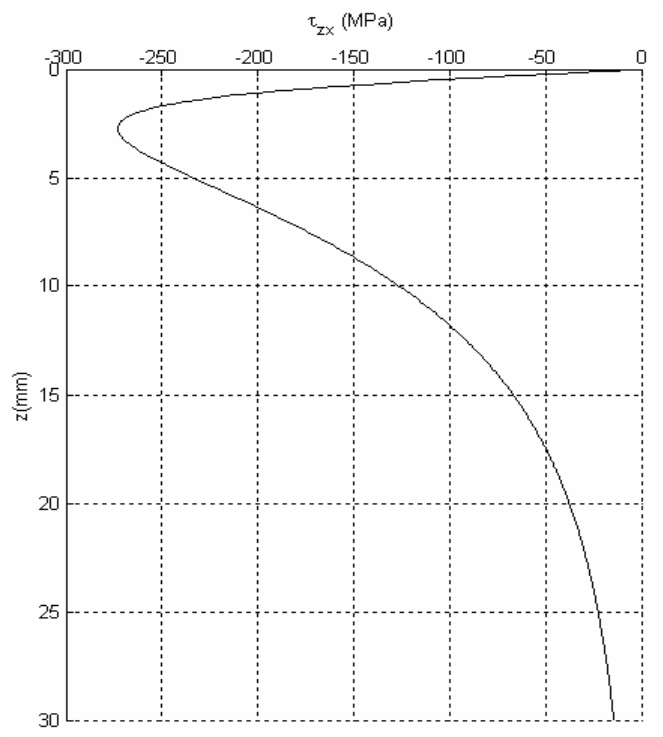

Figure 8. Shear Stress $\tau z x$ along the z-coordinated axle.

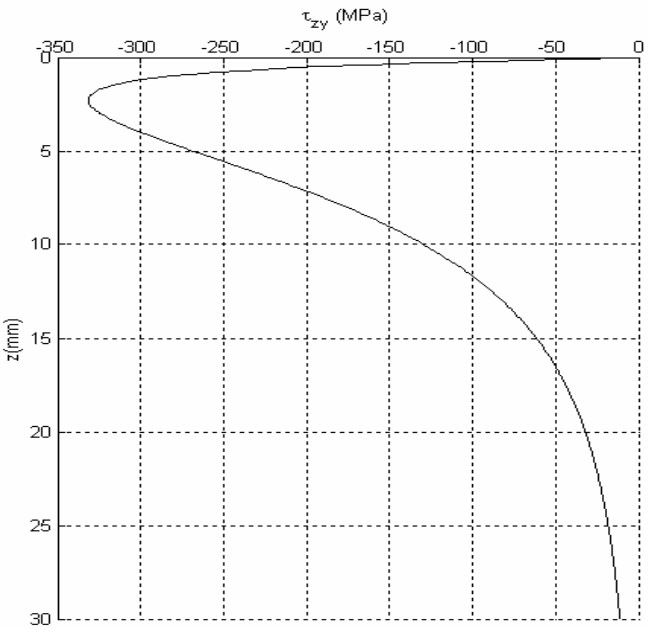

Figure 9. Shear Stress $\tau$ zy along the z-coordinated axle. 


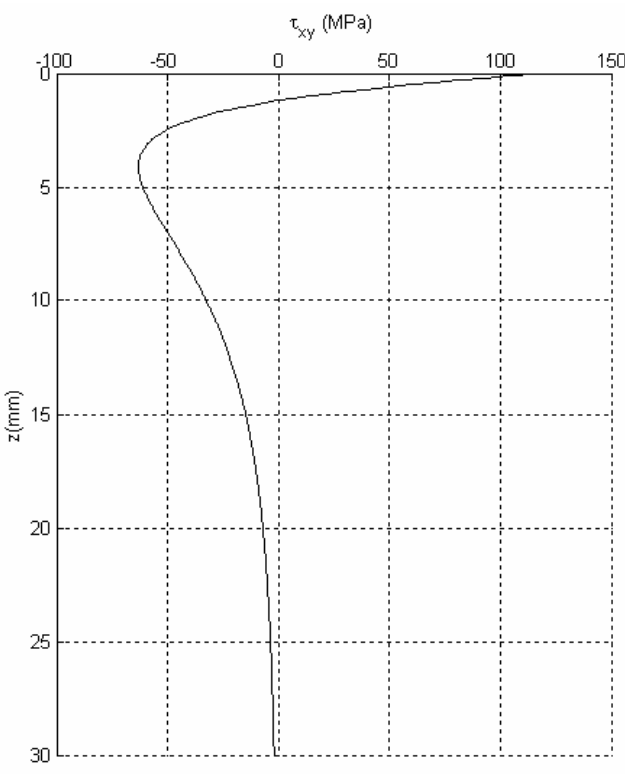

Figure 10. Shear Stress $\tau x y$ along the z-coordinated axle.

The order of magnitude of the maximum shearing stresses found is $350 \mathrm{MPa}$. The shearing stress endurance limit $\left(\mathrm{S}_{\mathrm{sn}}\right)$ may be estimated by multiplying the same multiplication factor used for the strain energy theory $(0,58)$ by the endurance limit. For an endurance limit of $1200 \mathrm{MPa}$, the value of $\mathrm{S}_{\mathrm{n}}$ is approximately $600 \mathrm{MPa}$ and the value of $\mathrm{S}_{\mathrm{sn}}$ may be estimated at about $350 \mathrm{MPa}$. In addition, one must consider the fact that the high temperatures caused by braking on the wheel itself significantly decrease the wheel material's endurance and that the inclusion of sulfites and other stress concentrating agents might be present (Santos, 1992). Although this is not the main criterion employed, it shows the importance of the presence of stresses with the magnitude found.

The values found for the stresses are extremely high when compared with the elastic limit of the type of steel usually employed in wheel manufacture. Nominal yield point values do not exceed $800 \mathrm{MPa}$. Thus, the elastic treatment used by all researchers cited, as well as in this study, must be the object of a new approach for the evaluation of loads carried on railroads similar to the ones used as examples of the use of this method. This line of research has been developed with the use of numeric tools (Santos and Santos, 2001).

The order of magnitude of actual subsurface shearing stresses, taking into consideration the initial yield under load, is certainly lower than the one shown in figures 5 to 7 . Nevertheless, with only one revolution under a high load, the whole tread of the wheel will have yielded. Due to the steel's own properties and since no significant inversion of loads is expected, the material will have a new distribution of stresses in the area near the surface. A traction load will occur, since, during the yield, the load that occurred was a compression load. Thus, in a shallow range, stress will vary between a significant amount of compression and a small amount of traction.

Below the surface high shearing stress will occur if compared with those on the surface, as shown in Fig. 6 and 7. According to studies on this issue (Gallagher et al., 1992; Santos, 1992), cracks originating from the surface might spread due to the existing traction, in a radial direction, along the " $\mathrm{z}$ " axle, and meet with those cracks that have started due to subsurface shearing. Due to the radial property of the crack which spreads through traction, this crack would only cause shelling if it reached the tangential crack caused by shearing. Otherwise, it would spread to wherever traction occurred and would stop at the subsurface compression area.
One of the methods used to evaluate the life in service of railroad wheels, calculated in terms of an equivalent shear stresses amount is the Dang Van criterion, which compares this amount to the shearing stress endurance limit. In order to determine the total amount of damage, a scan is performed at each point of the wheel tread and the estimated life under load is determinate. Even if there is previous residual tension - as long as it has occurred with no damage - the criterion may be employed. Thus, the work hereby described can be useful for the analysis of the life until failure occurs, since the previous distribution of stresses is known, and that may be combined with the one applied to the contact.

\section{Conclusions}

This study enabled us to calculate the order of magnitude of the expected elastic stresses and verify that the latter are severe enough to cause shelling. The study also enabled us to determine that, under high loading conditions, the likelihood of failure is very high, given the current characteristics of the railroads, as seen in the example of the Estrada de Ferro Carajás (Carajás Railroad).

The method used, based on Boussinesq potential functions and on the elastic half-space model, was instrumental for the evaluation of the stresses distribution and for comparison with the wheel material properties. With the results obtained, it was possible to identify the method's limitations when regard to the issue of high loads with bodies, which have the geometry of wheels, and rails. For the case in point, one must face the problem using an elastic-plastic model. This can be achieved based on the results of this study, by including the stresses distribution after the initial yield under a high load.

The study also shows an analytical manner of evaluating elastic stresses on contact bodies for the case in which the contact area is elliptic. Since compression yield occurs as soon as the railroad cars are loaded, and since iron ore carrying railroad cars seldom travel with loading conditions other than fully loaded or empty, one does not expect to find significant variances in the stresses caused by mechanical loads after the operation starts. Thus, the values found hereby could be combined with the distribution of stresses caused by the initial overload, and allow proper evaluation of the stresses that cause failure.

\section{References}

Carter, F. W., 1926, "On the action of a locomotive driving wheel". Proceedings of the Royal Society of London, London, v. 112, pp. 151-157.

Gallagher, M. T., Polzin, H. R.and Wetenkamp, H. R. Increased loading of 36 " wheels thermal and mechanical considerations. Association Annual Meeting, Chicago, Sep. 1992.

Haines, D. J. and Ollerton, E., 1963, "Contact stress distribution on elliptical contact surfaces subjected to radial and tangential forces". Proc. of Inst. of Mechanical Engineers, London, v. 177, n. 4, pp. 45-54.

Hamilton, G. M.and Goodman, L. E., 1966, "The stress field created by a circular sliding contact". Journal of Applied Mechanics, New York, v. 33, pp. 371-376.

Hertz, H. R., 1882, "Miscellaneous Papers". $8^{\text {th }}$ ed. London: Macmillan \& Co, 1896. Cap. 5 - "On the contact of two elastic solids", p. 146-162.

Johnson, K. L., 1985, "Contacts Mechanics". $1^{\text {st }}$ ed. Cambridge: Cambridge University Press, $451 \mathrm{pp}$.

Kalker, J. J., 1967, "On the rolling contact of two elastic bodies in the presence of dry friction", PhD Thesis, Department of Mechanical Engineering, Delft University of Technology, Delft, $155 \mathrm{pp}$.

Love, A. E. H., 1929, "The stress produced in a semi-infinite solid by pressure on part of the boundary". Phil. Trans. Royal Society, v. 228, pp. 377-420.

M'ewen, E., 1949, "Stresses in elastic cylinders in contact along a generatrix". Philosophical Magazine, Cambridge, v. 40, pp. 454-459.

Sackfield, A.; Hills, D. A., 1983, "Some useful results in the classical Hertz contact problem". Journal of Strain Analysis, London, v. 18, n. 2, pp. 101-105. 
Santos, A.A 1996, "Determinação do coeficiente global de transferência de calor" (In Portuguese), PhD Thesis, Faculty of Mechanical Engineer, State University of Campinas, Campinas, Brazil.

Santos, A.A., 1992, "Problemas causados pelo aquecimento em rodas ferroviárias - O ensaio Krauss". (In Portuguese), Master's Degree Dissertation, Faculty of Mechanical Engineer, State University of Campinas, Campinas, Brazil.

Santos, F. C., 2000, "Análise do contato roda - trilho e sua influência na vida em serviço de rodas ferroviárias”, (In Portuguese), Master's Degree Dissertation, Faculty of Mechanical Engineer, State University of Campinas, Campinas, Brazil, 151pp.
Santos, F. C. Santos Jr, A. A., 2001, "Análise elasto - plástica de rodas de vagões de carga utilizando o método dos elementos finitos”, XVI CONGRESSO BRASILEIRO DE ENGENHARIA MECÂNICA (COBEM),

Vol. 1, Uberlândia, Brasil, CD-ROM.

Smith, J. O.and Liu, C. K., 1953, "Stresses due to tangential and normal loads on an elastic solid with application to some contact stress problems". Journal of Applied Mechanics, New York, v. 20, pp. 157-166.

Stone, D. H., Moyar, G. L. and Guins, T. S., 1992, An interpretative review of railway wheel spalling and shelling. ASME RTD, v. 5, pp. 97-103. 